\title{
Research on Abnormal Noise Evaluation Method of Window Lift Motor Based on Subjective and Objective Psychoacoustics
}

\author{
Yi Zikui, Tan Jianping, Yan Tao, Liu Sisi \\ State Key Laboratory of High Performance Complex Manufacturing, Central South University, Changsha, China \\ Email address: \\ 18692200622@163.com (Yi Zikui),jptan@mail.csu.edu.cn (Tan Jianping), yantao_0421@163.com (Yan Tao), \\ 15200852420@163.com (Liu Sisi)
}

\section{To cite this article:}

Yi Zikui, Tan Jianping, Yan Tao, Liu Sisi. Research on Abnormal Noise Evaluation Method of Window Lift Motor Based on Subjective and Objective Psychoacoustics. International Journal of Mechanical Engineering and Applications. Vol. 4, No. 4, 2016, pp. 156-165. doi: $10.11648 /$ j.ijmea.20160404.14

Received: June 20, 2016; Accepted: July 23, 2016; Published: August 8, 2016

\begin{abstract}
To overcome the weakness of the existing noise evaluation method for the window lift motor, a kind of abnormal noise evaluation method based on objective psycho acoustic metrics is proposed. As the existing objective psycho acoustic metrics cannot be fully applicable to the window lift motor noise evaluation, based on the sharpness of the Zwicker model, a weighting factor similar to the window function used to modify the sharpness is proposed according to the characteristic of the window lift motor noise. The effectiveness of the objective metrics on the subjective perception is analysed by consistency experiment of the subjective and objective evaluation of the window lift motor noise. The results show that the loudness and modified sharpness have strong correlation with the subjective evaluation results and can accurately reflect the subjective feelings of people. So they can be used for window lift motor noise classification and evaluation.
\end{abstract}

Keywords: Window Lift Motor Noise, Modified Psycho Acoustic Parameter, Subjective and Objective Evaluation

\section{Introduction}

With the development of the economy and the automobile industry, requirements on the driving and riding comfort continue to raise. The noise generated in the operation of the window lift motor which is close to the driver and passengers has psychological impact on them, and thus leads to a reduced driving and riding comfort. Therefore, the motor noise inspection must be carried out before delivery.

According to the national standard GB/T 18697-2002, the A-weighted sound pressure level evaluation method is proposed and widely used as a domestic motor noise inspection method. But the use of A-weighted sound pressure level evaluation method has the following disadvantages as indicated by the literatures $[1,2]:[1]$ states that description on the low-frequency noise is inaccurate; [2] cannot describe the difference between steady and intermittent noise. So a motor comfort to the A-weighted sound pressure level still may bring a unpleasant noise and the A-weighted sound pressure level evaluation method cannot be used as an effective standard to evaluate the window lift motor noise. In addition to the A-weighted sound pressure level, another frequently used method is the spectrum analysis method. But the spectrum analysis method is limited on the computation of "sound" as a kind of physical phenomenon, while the actual sound events is an auditory perception process and the human being is the judger. So the spectrum analysis method cannot accurately reflect the irritable degree of noise. And it leads to a great limitation on practical applications [3]. Therefore, the subjective evaluation method on noise evaluation of window lift motor is still popular in actual manufacturing process. The advantage of this method is that the judgement is made according to human being's real feelings. But as all known, the subjective evaluation method has the disadvantages of poor consistency of the evaluation results, risks of incorrect judgement and skipped judgment.

At present, based on psychoacoustic point of view, many 
scholars have attempted to use objective psychoacoustic metrics [4-7] as characteristic values to evaluate and detect the vehicle interior noise, engine radiation noise and so on, which show a good application effect. As the evaluation of the window lift motor noise also needs to describe the subjective feelings of human being by the measurable objective parameters, the same practice can be used for this evaluation. Currently, four parameters of loudness, sharpness, roughness, fluctuation are the mostly popular objective psychoacoustic metrics, and a variety of calculation models have been proposed by researchers worldwide [8-10]. But, those objective psychoacoustic models are not applicable to all types of noise [11]. For the window lift motor noise evaluation, usage of those metrics without modification will lead to an inaccurate description of human being's subjective feelings. Therefore, it is necessary to modify those metrics according to the characteristics of the sound signal of the window lift motor, and to verify the validity of the objective parameters by the subjective evaluation experiments.

\section{Subjective and Objective Evaluation Principle of Noise from the Window Lift Motor}

Abnormal noise from the window lift motor can be roughly divided as brush-commutator noise, worm-gear noise, bearing noise and gap noise according to the statistical data of a window lift motor manufacturer. Thereof, brushcommutator noise and worm-gear noise are the main types of abnormal noise, accounting for around 94\% of the total number of abnormal noise. In order to improve the current noise evaluation method in the factory and overcome the weakness of traditional psychoacoustic metrics, the acceptable noise, brush-commutator noise, worm-gear noise from window lift motors provided by a motor manufacturing factory are taken as research objects, and a model of applicable and modified objective psychoacoustic metrics is proposed in this article by using subjective and objective evaluation methods according to the characteristic of the window motor noise.

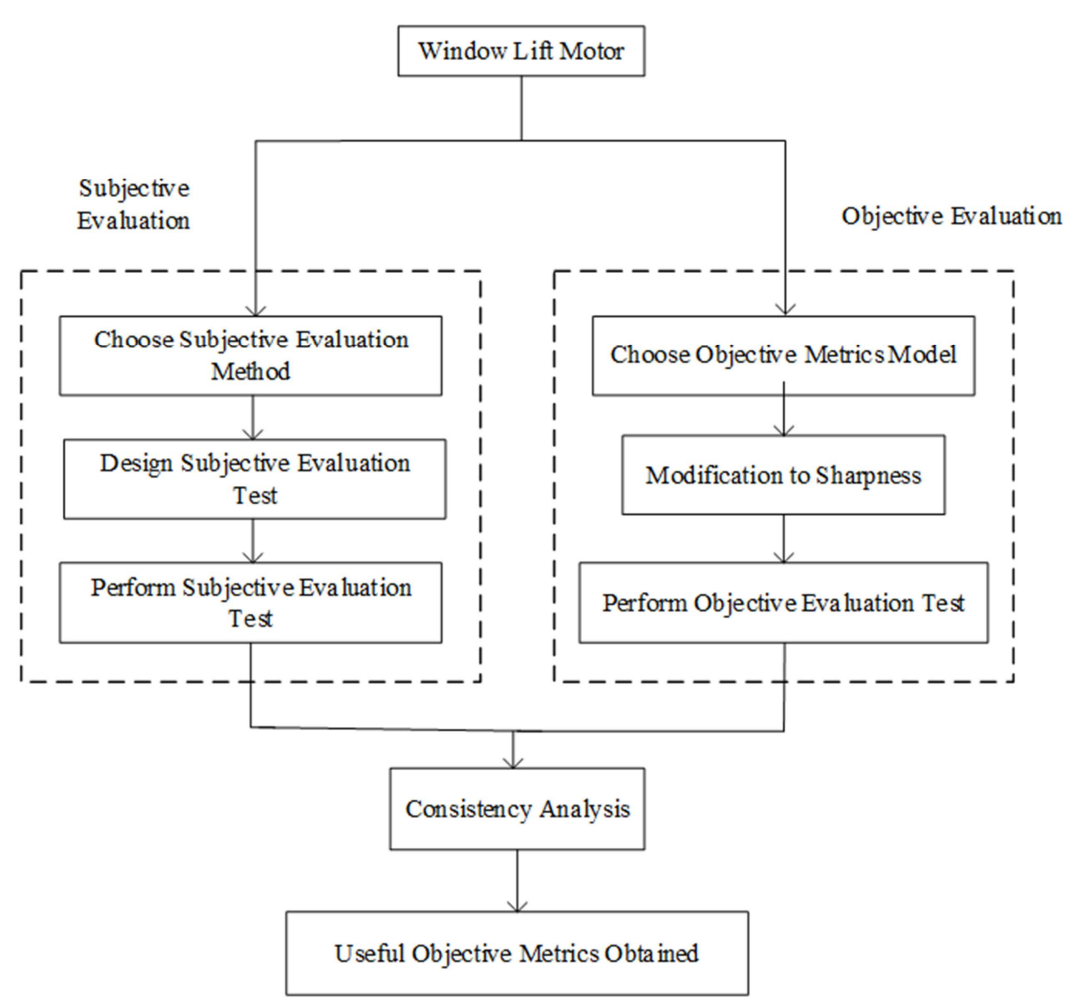

Fig. 1. Flow chart of the subjective and objective evaluation of noise from window lift motor.

Figure 1 shows the window lift motor noise subjective and objective evaluation process, which can be roughly divided into the following three parts:

(1) Objective evaluation:

Based on the calculation model of objective psychoacoustic metrics, the objective metrics of the noise from the window lift motor are calculated according to the noise characteristic of the window lift motor.

(2) Subjective evaluation:

At first, the appropriate subjective evaluation method is applied and the subjective evaluation experiment is designed, then the subjective evaluation results of the noise from the window lift motor are obtained by the subjective evaluation experiments;

(3) Consistency analysis of subjective and objective evaluation:

The consistency of subjective evaluation results and objective evaluation results are analysed to estimate the relevance between each other. According to the value of correlation coefficients, the effectiveness of objective metrics 
on description of the subjective feelings is figured out.

\section{Study on the Objective Evaluation of Motor Noise}

As the window lift motor noise samples are measured in the factory anechoic chamber, the Zwicker loudness model which is suitable to free acoustic field is used. The loudness calculation is made on Zwicker loudness program specified by ISO 532B and DIN 45631 [12]. According to the literature [13], if the Zwicker loudness model is applied, the Zwicker sharpness, roughness and fluctuation model [14] should be also considered. So, we choose Zwicker model for calculation of all metrics in this article.

\subsection{Loudness Model}

In Zwicker model, the loudness is obtained by calculation of specific loudness in each critical band. The specific loudness stands for the loudness of noise in a band, which can be obtained by the calculation of stimulating stage E. Formula 1 is the main loudness calculation formula. The specific loudness is constituted by main loudness added with slope loudness according to the DIN 45631.

$$
N_{s}=N_{0}^{\prime}\left(\frac{E_{T Q}}{s E_{0}}\right)^{k}\left[\left(1-s+\frac{s E}{E_{T Q}}\right)^{k}-1\right]\left(\frac{\text { Sone }}{\text { Bark }}\right)
$$

Thereof, $E_{T Q}$ is quiet threshold stimulating stage; $E_{0}$ is the stimulating stage when reference sound intensity $I_{0}=10^{-12} \mathrm{~W} / \mathrm{m}^{2} ; N_{0}^{\prime}$ is the reference specific loudness, when it is 0.08 , then $\mathrm{s}=0.5$ and $\mathrm{k}=0.23$, and when it is 0.0635 , then $\mathrm{s}=0.25$ and $\mathrm{k}=0.25$. The stimulating stage $E$ is the sound pressure level of critical band after the sound transfer through the middle ear.

The overall loudness $N$ is calculated by integral of specific loudness in the whole band domain.

\subsection{Roughness Model}

From the Hilbert Demodulation on window lift motor noise, the sound signal modulation frequency of the accepted noise and the brush-commutator noise stay in the vicinity of $100 \mathrm{~Hz}$, the sound signal modulation frequency of the wormgear noise stay in the vicinity of the $35 \mathrm{~Hz}$, and all sound signal modulation frequency stay above $20 \mathrm{~Hz}$. According to the definition of roughness and fluctuation [14], the noise of the window lift motor can be described by roughness rather than fluctuation.

The roughness calculation is based on the Zwicker model, whose formula is as follows:

$$
R=0.3 f_{\bmod } \int_{0}^{24 b a r k} \Delta L_{E}(z) d z
$$

Thereof $R$ is roughness, $f_{\text {mod }}$ is the modulation frequency,
$\Delta L_{E}(z)=20 \log _{10}\left(\frac{N_{\max }^{\prime}}{N_{\min }^{\prime}}\right)$ is the variation of incentive level.

\subsection{Sharpness Model Modification}

The sharpness reflects the feeling of shrill and piercing noise. According to the Zwicker sharpness model, the formula (3) is used to calculate the sharpness.

$$
S=C \frac{\int_{0}^{24 b a r k} N_{s}(z) \cdot Z \cdot g(z) d z}{N}
$$

Thereof, $S$ is sharpness, $N$ is overall loudness, $C=0.22$ is ratio coefficient, $z$ is critical band rate, $N_{s}$ is specific loudness. $g(z)$ is weighting factors, currently following 3 kinds of weighting factors are mainly used[15-18].

$$
\begin{gathered}
g(z)= \begin{cases}1 & \\
0.00012 z^{4}-0.0056 z^{3}+0.1 z^{2}-0.81 z+3.51 & z>14\end{cases} \\
g(z)= \begin{cases}1 & z \leq 16 \\
e^{0.5 \bullet \ln (2) \bullet(z-16)} & z>16\end{cases} \\
g(z)= \begin{cases}1 & z \leq 16 \\
0.066 e^{0.171 z} & z>16\end{cases}
\end{gathered}
$$

Taking sound signal from window lift motors as the research object, no matter which kind of weighting factor is selected, sharpness calculation according to the formula (3) is always inconsistent with the subjective perception, as the sharpness metrics of obvious shrill noise samples are basically the same as that of accepted noise samples (shown as Figure 2). Therefore, it is necessary to modify the Zwicker sharpness model according to the characteristic of the noise from the window lift motor.

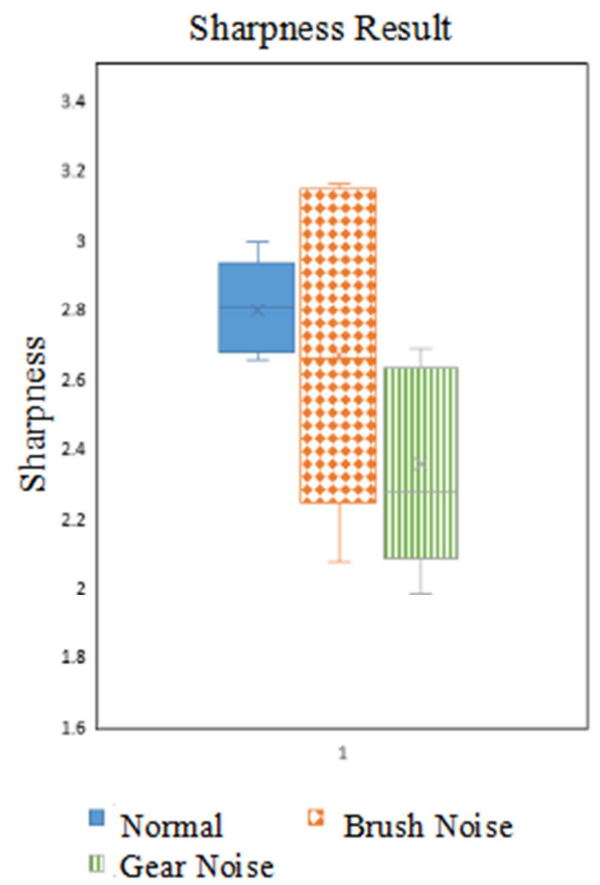

Fig. 2. Zwicker sharpness calculation results (Weighting factor as formula 6). 
Zwicker sharpness calculation model is showed as formula 2. According to the mathematical understanding of the model, the sharpness basically standards for the result of data normalizing of specific loudness's weighted first moment. Thereof, the normalizing process is used to eliminate the differences caused by different loudness from variant sound samples, and the weighting factor is used to emphasize the contributing components from the specific loudness which reflect the feeling of "shrill".

Specific loudness $N_{s}$ of 7 accepted motor noises, 15 brush-commutator motor noises, 10 worm-gear motor noises are analysed.

At first, specific loudness of all sound samples are calculated and normalized. The normalizing process shows as formula 7 .

$$
N_{s}^{\prime}=\frac{N_{s}}{N}
$$

Thereof, $N_{s}$ ' is the specific loudness after normalization, $N_{s}$ is original specific loudness, $N$ is overall loudness.

Then calculate the arithmetic average value of normalized specific loudness on each motor category. Each average value stands for specific loudness of the corresponding category. After mapping and analysis of these three kinds of normalized specific loudness, it can be concluded that: specific loudness of the brush-commutator noise and the worm-gear noise are significantly higher than that of accepted noise in around $2 \sim 10$ barks of the threshold band rate, and there's no such obvious difference in other threshold band rates. The normalized specific loudness curves are showed as Figure 3.

Therefore the specifics of shrill from the brushcommutator noise and the worm-gear noise can be assumed to be caused by the difference in $2 \sim 10$ Barks. According to this hypothesis, the weighting factors in formula 4 to 6 are unable to emphasize the contribution of specific loudness on shrill feelings in $2 \sim 10$ Barks. Therefore, a new weighting factor $g(z)$ (shown as formula 8), which is similar to window function, is proposed to modify the sharpness model in order to emphasize the contribution of this component to the sharpness. Finally, the value of the integer ' $a$ ' in the weighting factor of formula 8 is obtained and the validity of this amendment is verified by the objective and subjective evaluation experiment.

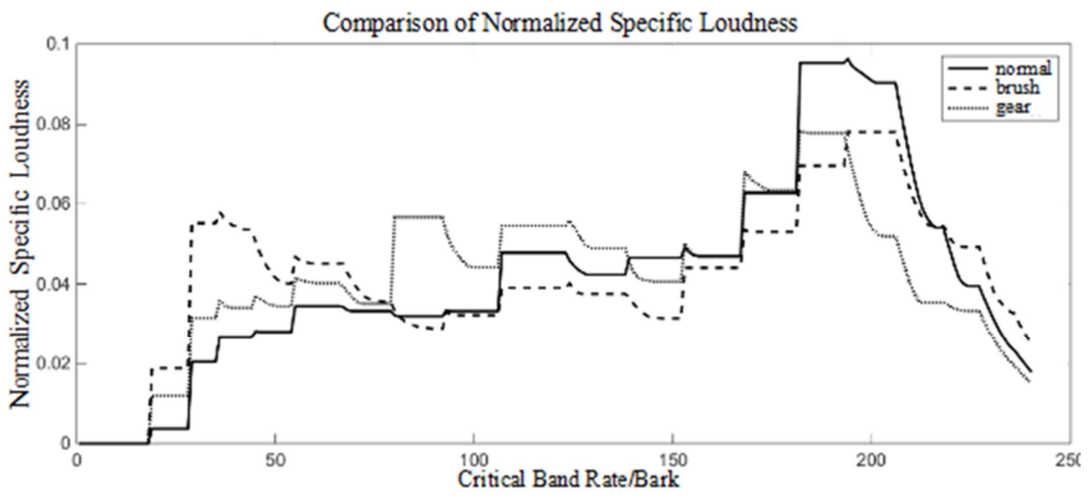

Fig. 3. Normalized specific loudness comparison chart between accepted motor and fault motors.

$$
g(z)= \begin{cases}1 & z<2 \\ a & 2 \leq z \leq 10 \\ 1 & z>10\end{cases}
$$

\section{Study on Subjective Evaluation of Motor Noise}

\subsection{Subjective Evaluation Method of Psycho Acoustics}

Subjective evaluation is used to judge whether the subjective feelings of the motor noise can be accurately described by objective metrics, which needs to be studied in a variety of voice properties of a large number of samples. According to the commonly used methods introduced in the literature [19]' the semantic differential method is used in this article for subjective evaluation.

The semantic differential method provides some polar adjectives as evaluation criteria, and then sets up a number of class intervals for evaluation. Due to the reasons that the evaluation scale changing continuously, extreme values may not being selected and psychological expectations affecting assessors in the semantic differential method assessment, it easily leads to a poor consistency and concentration in a small range of evaluation results. To overcome the weakness, anchored semantic differential method [7] with extreme scores sample is applied in the experimental design. The neutral samples are selected as reference samples and the polar semantics samples are selected as extreme samples. Those samples have been played back in the beginning of the evaluation process as the calibration and then assessor give semantic evaluation score to testing samples.

\subsection{Design of Subjective Evaluation Experiment}

Based on the semantic differential method, this paper carries out the subjective evaluation of the experimental process designed as follows: 


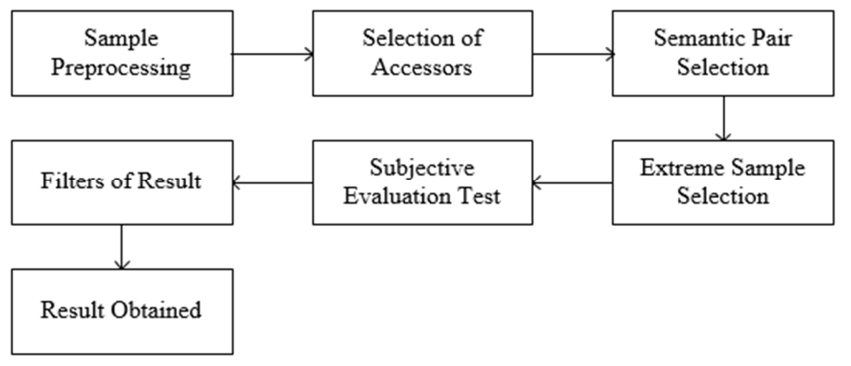

Fig. 4. Experimental process of subjective evaluation.

The process in Figure 4 is shown as below:

(1) Sample preprocessing: to remove the part with poor quality in the sound samples, and to uniform sound samples with the same length;

(2) Selection of Assessor: the subjective experience of the noise of the window lift motor is the common sense of normal drivers and passengers, so the assessor can be defined as ordinary people;

(3) The semantic pair selection: the semantic pair should be selected according to the subjective perception of the objective parameter, which is generally accepted by the assessor;

(4) Extreme samples selection: for each semantic pair, select the sample corresponding to the extreme value based on the anchored semantic differential method, in order to implement the subjective evaluation afterwards;

(5) Subjective evaluation experiment: using the anchored semantic differential method with extreme value samples to carry on the subjective evaluation experiment;

(6) Filter of the evaluation results: for each semantic pair, the correlation coefficients among the evaluation results of every assessor were calculated and the result of the assessor who get the low coefficient were excluded.

(7) Acquisition of the subjective evaluation results for each semantic pair: For each semantic pair, take the arithmetic mean of evaluation results after filters as the subjective evaluation results of the semantic pair.

\section{The Consistency Experiment of Subjective and Objective Evaluation}

\subsection{Subjective Evaluation Experiment}

\subsubsection{Experimental Objective}

According to the subjective evaluation experiment process in Figure 4, take semantic pair of "loud - soft", "shrill - dull" and "noisy - clear", which are corresponding to the objective metrics of loudness, sharpness and roughness, to evaluate the noise of window lift motor with type "FPC3 12V F00S1W2 014". The supporting data for the further subjective and objective analysis is obtained by subjective evaluation experiment which evaluate the feeling difference with different kind of motors.

\subsubsection{Experimental Condition}

(1) Sample preprocessing methods: take a total of seven accepted motor noise, 15 brush-commutator motor noise and 10 worm-gear motor noise as the sound samples, and then remove the sound fragment which is seriously polluted by the speaking voice. After operation on equipment with software of CoolEdit Pro 2.1, sound signals with length of 4.5 seconds are finally obtained;

(2) Assessor: the assessor team is setup with 7 females and 10 males with age range of $21 \sim 23$ years old and without hearing system diseases;

(3) Software environment: Based on MATLAB R2014b under Windows Microsoft 10 64bit, develop new GUI to carry out extreme sample selection and anchored semantic differential evaluation.

Extreme sample selection GUI is showed as Figure 5. At first, assessors listen to samples by clicking button of "samples A play" and "sample B play" separately, and then select the corresponding options according to the subjective evaluation results. All sample evaluation results can be checked by clicking button of "completed, view result".

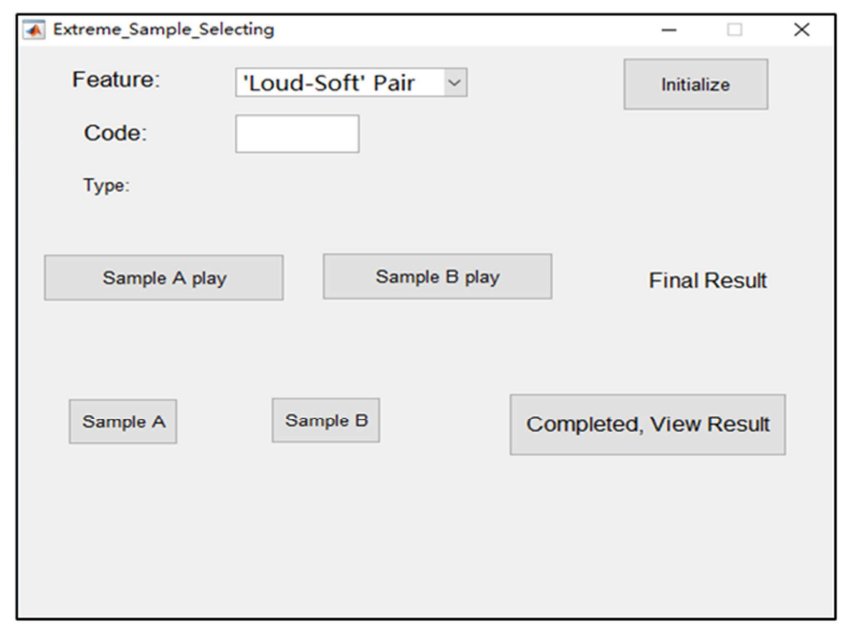

Fig. 5. Extreme sample selection interface.

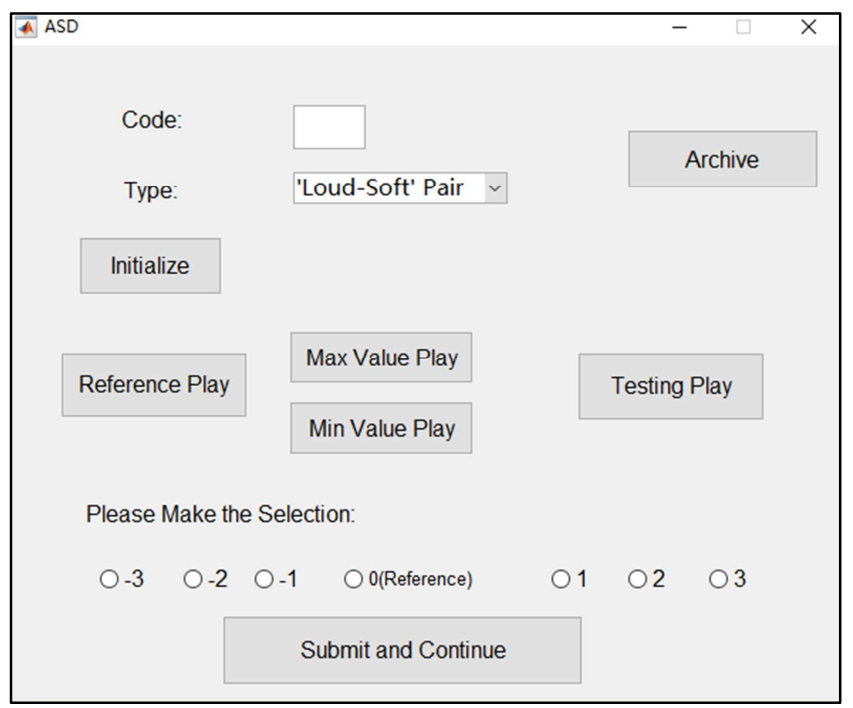

Fig. 6. Evaluation interface of the anchored semantic differential method.

Anchored semantic differential evaluation GUI is shown as 
Figure 6. For each semantic pair, assessors are calibrated with reference samples and extreme samples firstly before judgment. Take the semantic pair of "loud - soft" as an example, score of "-3" represent the most soft sample, and score of " 3 " represent the most loud sample. After all sample evaluations are completed, click the "archive" button to generate the data file of "evaluation type - the evaluation of assessor number.xlsx".

(4) Hardware environment: the subjective evaluation experiment is carried on the laptop, whose hardware configuration is: Intel (R) Core (TM) i5-3210M CPU, 4GB RAM. Sound play device is 1MORE high fidelity headphone.

(5) Site environment: the experiment was conducted in a quiet room with a background noise of $37 \mathrm{db}$.

\subsubsection{Experimental Result Analysis}

The correlation coefficients of the evaluation results from different assessors are obtained by the subjective evaluation experiments, shown as Figure 7 9. The assessor numbers are the horizontal and vertical coordinates, and the color brightness represent the correlation coefficients among different evaluation results from different assessors. According to Figure 7 9, following conclusions can be obtained.

(1) According to the evaluation experiment of "loud - soft" semantic pair, the correlation coefficients among different evaluation results were high and there's no poor evaluation result from all assessors. The subjective evaluation results can be achieved by average of all evaluation results.

(2) According to the evaluation experiment of "shrill dull" semantic pair, the evaluation results of No.2 and No. 8 assessor should be filtered out as their evaluation results have relatively low correlation coefficients with others. The subjective evaluation results can be achieved by average of all remaining results.

(3) According to the evaluation experiment of "noisy clear" semantic pair, the value of correlation coefficient is generally below 0.6. The correlation is low, so as the effectiveness of the evaluation result. Therefore, the semantic pair of "noisy - clear" cannot reflect the motor noise effectively, and the corresponding objective parameter of roughness cannot be effectively used for the evaluation of motor noise as well.

The following two factors lead to the low effectiveness of "noisy - clear" semantics pair: (1) it's difficult for the assessor to achieve the right judgment as the noise characteristics of "noisy - clear" from window lift motor is not obvious; (2) the evaluation results have great volatility as difference of the noisy feeling strength differences among assessors is very big.

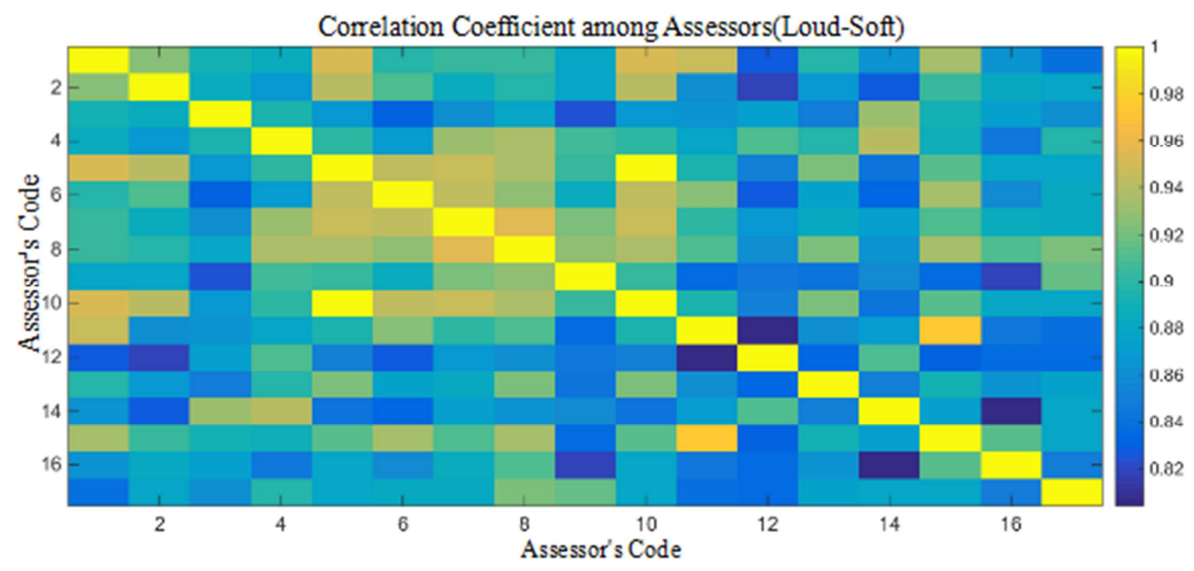

Fig. 7. The correlation coefficient of assessors in l'oud and soft'semantic pair.

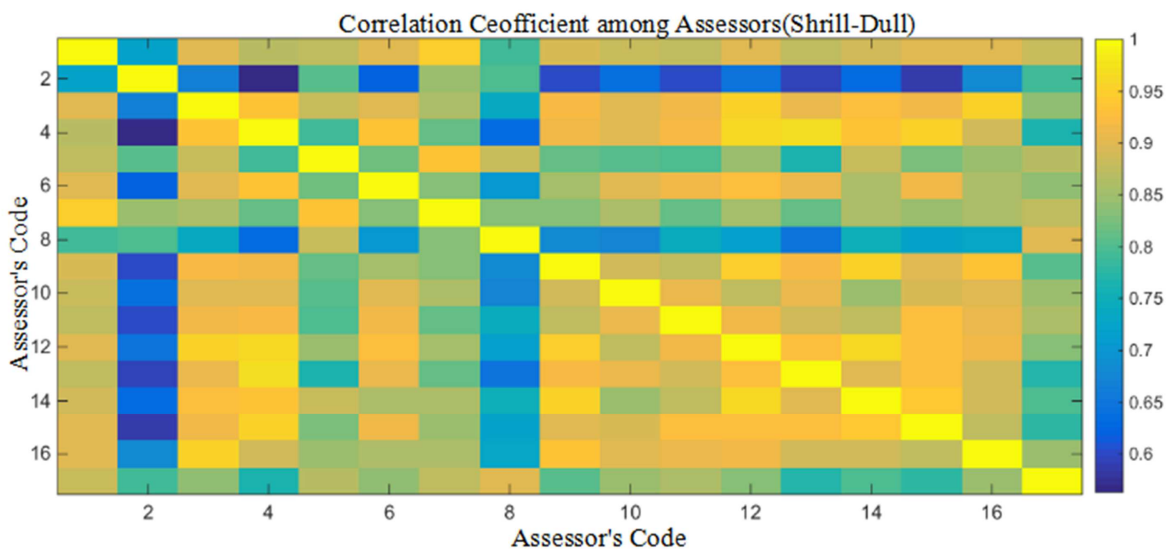

Fig. 8. The correlation coefficient of assessors in shrill and dull'semantic pair. 


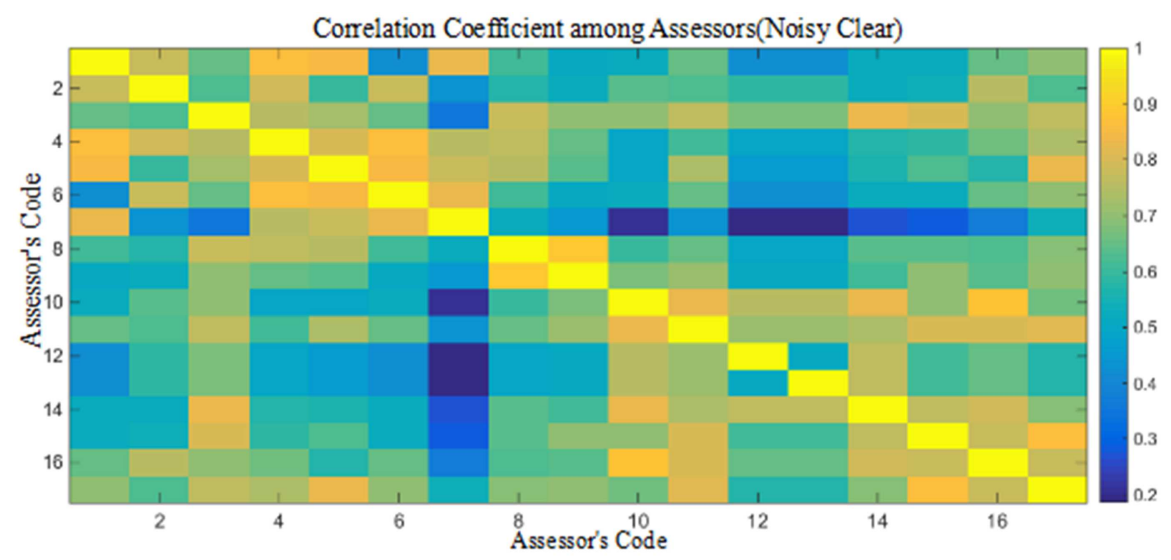

Fig. 9. The correlation coefficient of assessors in hoisy and clear"semantic pair.

The subjective evaluation results of "loud - soft" semantic pair and "shrill - dull" semantic pair are shown as shown Figure 10. It can be seen that, compared with accepted motor noise, the brush-commutator noise and the worm-gear noise bring significant "loud" and "shrill" feelings to assessors.
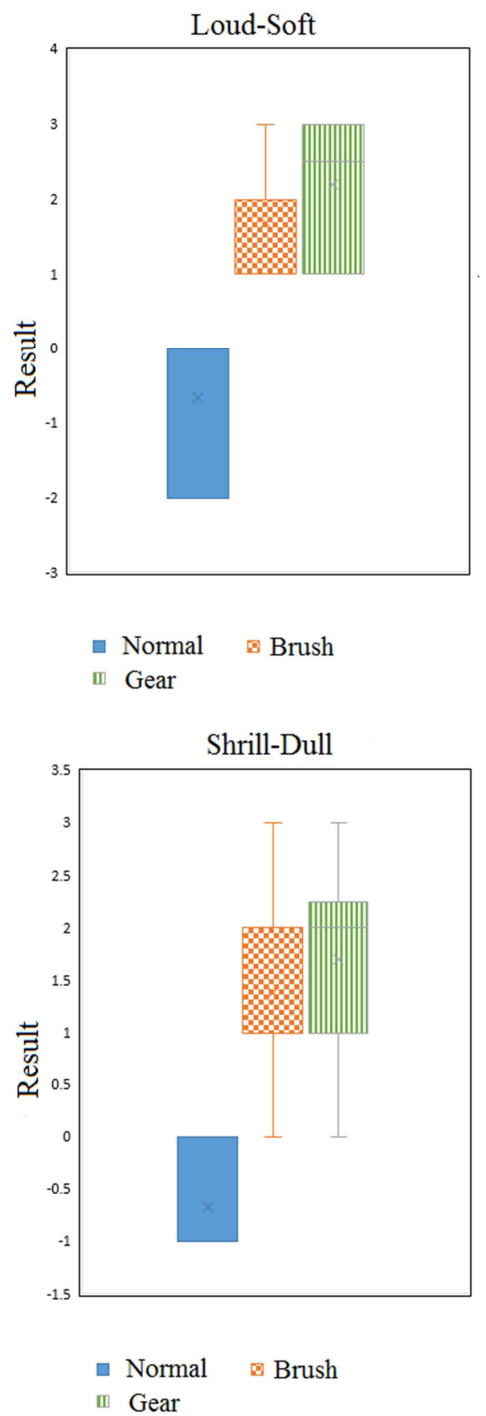

Fig. 10. Subjective evaluation results (left: l'oud - soft"semantic pair; right: shrill-dull'semantic pair).

\subsection{Objective Evaluation Experiment}

\subsubsection{Experimental Objective}

According to the conclusion from the subjective evaluation experiment, ignore roughness and take loudness and sharpness as objective metrics for calculation. The objective evaluation result will be used for further subjective and objective analysis.

\subsubsection{Experimental Condition}

(1) Sample selection: select 32 window lift motor noise from the subjective evaluation experiment for objective evaluation.

(2) Software environment: calculate based on software of Windows MATLAB R2014b.

\subsubsection{Experimental Result Analysis}

Objective evaluation results from the calculation of the loudness and sharpness parameters are shown as Figure $11 \sim 14$. In the sharpness calculation, set the value of the modified weighting factor in formula 8 as 2, 4, 6, 8, 10 and 12 respectively.

Calculation results of loudness and sharpness are shown as follows:

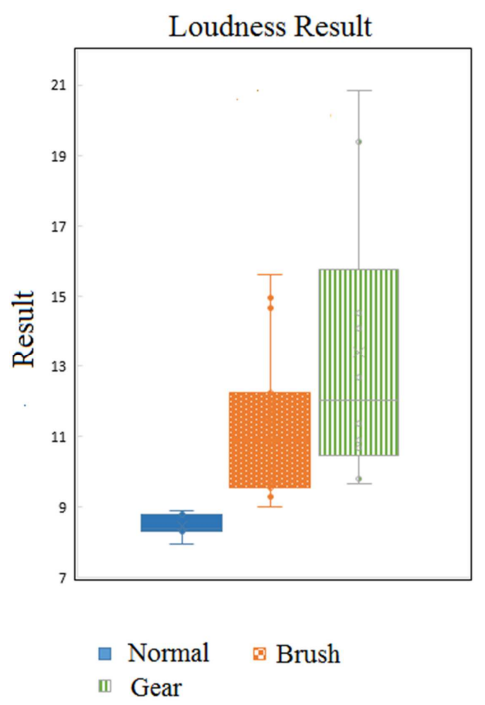

Fig. 11. Calculation result of loudness. 


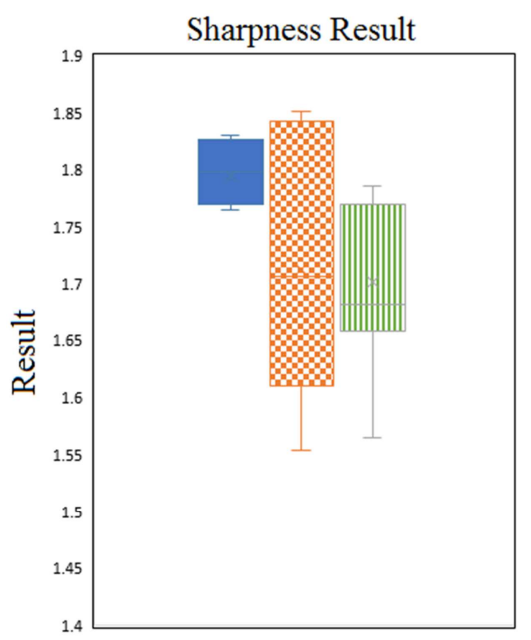

- Normal Brush

I. Gear

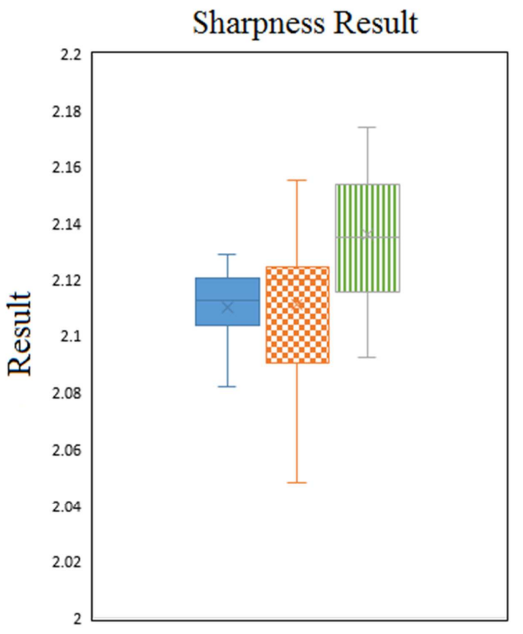

- Normal Brush

w Gear

Fig. 12. Calculation result of modified sharpness (left: $a=2$; right: $a=4$ ).

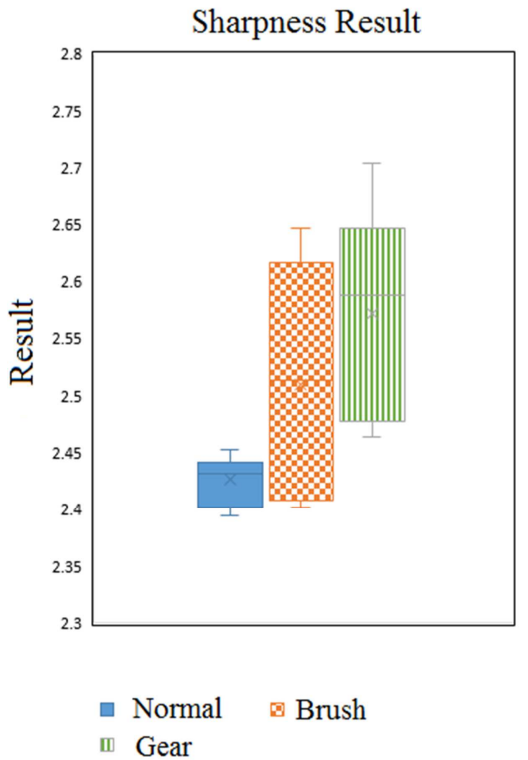

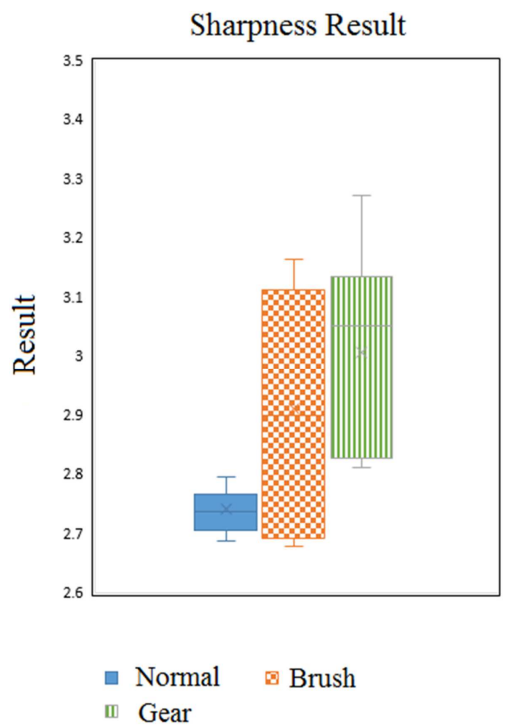

Fig. 13. Calculation result of modified sharpness (left: $a=6$; right: $a=8$ ).

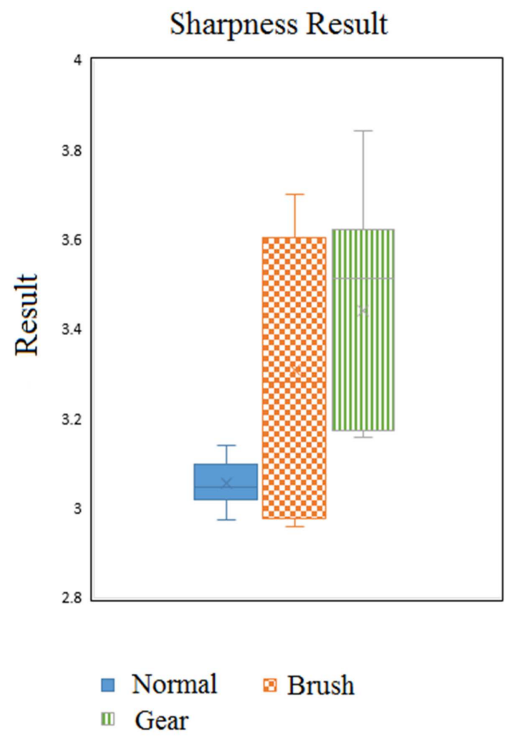

Sharpness Result

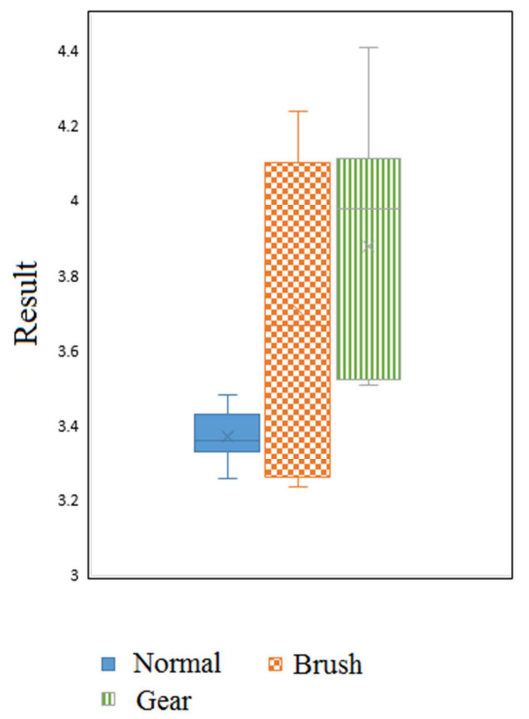

Fig. 14. Calculation result of modified sharpness (left: $a=10$; right: $a=12$ ). 
The conclusions from above calculation results are:

(1) The loudness calculation results of most of the accepted motors were significantly lower than that of those motors with the two failure types. Only limited amounts of accepted motor's loudness results are close to that of the failure motors.

(2) Based on sharpness model with the modified weighting factor, when a is greater than or equal to 6 , sharpness calculation results from three different kinds of motors have obviously difference and the sharpness of the accepted motors is lower than that of the other two kinds of failure motors.

\subsection{Subjective and Objective Evaluation Analysis}

The correlation coefficients between the subjective evaluation results and the corresponding objective metrics are calculated and the results are shown as Table 1:

Table 1. Correlation coefficient of subjective and objective evaluation results.

\begin{tabular}{lllllll}
\hline Loudness & Sharpness $(\mathbf{a}=\mathbf{2})$ & Sharpness $(\mathbf{a}=\mathbf{4})$ & Sharpness $(\mathbf{a}=\mathbf{6})$ & Sharpness $(\mathbf{a}=\mathbf{8})$ & Sharpness $(\mathbf{a}=\mathbf{1 0})$ & Sharpness $(\mathbf{a}=\mathbf{1 2})$ \\
\hline 0.7438 & -0.6007 & 0.1849 & 0.7221 & 0.7139 & 0.7113 \\
\hline
\end{tabular}

(1) The correlation coefficient between loudness and "loud - soft" semantic pair is 0.7438. There's a strong correlation between subjective and objective evaluation results. Therefore, loudness metrics can be used to accurately describe the subjective feeling of window lift motor noise.

(2) When a is greater than or equal to 6 , the modified sharpness metrics has strong correlation with the subjective feelings. So it can be also used to accurately describe the subjective feelings of the window lift motor noise. Furthermore, the correlation was highest when $a=6$, so set the value of a as 6 in the formula 8 .

\section{Conclusion}

(1) Taking the window lift motor of type "FPC3 $12 \mathrm{~V}$ F00S1W2 014" as the study object, the paper proposed a kind of abnormal noise evaluation method based on objective psychoacoustics metrics. And according to the characteristic of the motor noise, a new weighting factor to modify the sharpness parameter is proposed. From the experiment results of subjective and objective evaluation, the correlation coefficient of loudness and subjective feelings of "loud soft", and the correlation coefficient of modified sharpness and subjective feelings of "shrill - dull" is above 0.7. So they have strong correlation between each other, and the objective metrics of loudness and modified sharpness can accurately reflect the subjective feelings and can be suitable for evaluation of window lift motor noise.

(2) According to the characteristic of the fault motor noise, the paper proposed a new weighting factor similar to window function to modify Zwicker sharpness models. Based on subjective and objective evaluation and analysis results, set the value of weighting factor as 6 as the correction between the subjective and objective evaluation is the highest of 0.7221 , which means that the modified sharpness parameter can effectively reflect the subjective feeling.

(3) According to the characteristic of the window lift motor noise and subjective evaluation requirement, a kind of subjective evaluation experiment of anchored semantic differential method with extreme samples is designed. The characteristic of "loud" and "shrill" in the noise of failure window lift motors and subjective evaluation results of different samples are obtained by the subjective evaluation experiment, which provides a reference for the selection and correction of the objective parameters.

\section{References}

[1] Ute J. Meaning in the context of sound quality assessment [J]. Acta Acustica united with Acustica, 1999, 85(5): 679-684.

[2] Winfried K. Sound quality evaluation in the product cycle [J]. Acta Acustica united with Acustica, 1997, 83(5): 784-788.

[3] Zhang Wei, Jiang Weikang. Identification of Abnormal In-car Noise Based on Psychoacoustics Analysis [J]. Automotive Engineering: 2003, 25(6): 603-605.

[4] Un-Chang Jeong, Jin-Su Kim, Jae-Eun Jeong, In-Hyung Yang, Jae-Eung Oh. Development of a sound quality index for the wash cycle process of front-loading washing machines considering the impacts of individual noise sources $[\mathrm{J}]$. Applied Acoustics. 2015, 87: 183-189.

[5] Y. S. Wang, G. Q. Shen, Y. F. Xing. A sound quality model for objective synthesis evaluation of vehicle interior noise based on artificial neural network [J]. Mechanical Systems and Signal Processing. 2014, 45: 255-266.

[6] Gang-Ping Tan, Deng-Feng Wang, Qian Li. Vehicle Interior Sound Quality Prediction Based on Back Propagation Neural Network [J]. Procedia Environmental Sciences. 2011. 11:471477.

[7] Ricardo Penna Leite, Stephan Paul, Samir N. Y. Gerges. A sound quality-based investigation of the HVAC system noise of an automobile model [J]. Applied Acoustics. 2009, 70:636645 .

[8] Jiao Zhongxing. The Algorithm and Realization of Psychoacoustic Metrics [D]. Wuhan: Huazhong University of Science and Technology, 2011.

[9] DIN45631: 1991, Program for Calculating Loudness. Deutsche Industrie Normen. 1991,03.

[10] ISO 532-1975 Acoustic-Method for calculating loudness level [S].

[11] MAO Dong-xing. Progress in sound quality research and application [J]. Technical Acoustics. 2007, 26(1): 159-164. 
[12] Zwicker E, Fastl H, Widmann U,et al. Program for calculating loudness according to DIN45631 (ISO 532B) [J]. Journal of the Acoustical Society of Japan, 1990, 12(1): 39-42.

[13] Sun Huihui. Research on Evaluation of vehicle sound quality based on GA-BP neural network. [D]. Changchun: Jilin University, 2012.

[14] Fastl H, Zwicker E. Psychoacoustics Facts and Models [M]. Berlin Heidelberg New York: Springer, 2007.

[15] ZHAO Zhong-feng, CHEN Ke-an. Objective Evaluation Approaches for Noise Based on Zwicker Theory. [J]. Audio Engineering, 2005(12): 68-70.

[16] Tao Jianxing, Ding Houming, Yang Shengmei. Development and Application of Techniques for Evaluating Sound Quality of Air-conditioners [J]. Journal of Vibration, Measurement\& Diagnosis, 2001(3): 215-219.

[17] WANG Na,CHEN Ke-an. Application of Specific-loundnessbased Psychoacoustic Features in Automobile Recognition [J]. Audio Engineering, 2006(6): 34-36.

[18] Shiyan, Bi Fengrong, Lin Manqun, Jing Yabing et al. Basic Phychoacoustics Theoretics and its Application in Soundquality Evaluation [J]. SMALL INTERNAL COMBUSTION ENGINE AND MOTORCYCLE, 2006(1): 57-60.

[19] Liu Ningning. Study On Subjective Evaluation Method For Comprehensive Annoyance Of Vehicle Interior Noise [D]. Shanghai: Shanghai University of Engineering Science. 2013. 